\title{
TRADUCTION DES NOMS DES FILMS FRANÇAIS : ASPECT LINGUOCULTUREL
}

\author{
Viktoriia Kulykova \\ Candidat ès lettres, maître de conférences, maître de conférences du département de théorie, \\ pratique et traduction de la langue française, \\ Université technique nationale de l'Ukraine \\ "Institut polytechnique de Kyiv Igor Sikorsky", Ukraine \\ e-mail: koul.kiev@ukr.net, orcid.org/0000-0002-4155-6262
}

\section{Résumé}

L'article proposé étudie les moyens de traduction en ukrainien des particularités linguistiques et culturologiques des noms des films français. En particulier, on détermine les caractéristiques lexicales et sémantiques des titres français et les voies de leur reproduction dans la langue cible à travers le prisme de l'approche culturologique. Les titres représentant une situation verbalement codée qui est modélisée par le film se concentrent sur l'attention particulière du spectateur. A cette fin, ils sont brillants, attirants, expressifs. Les fonctions principales des noms des films sont les suivantes: nominative, c'est-à-dire la présentation au spectateur de l'idée principale du film; informative, à savoir la transmission des informations principales; publicitaire, pour attirer le public; pronostique, c'est-à-dire l'addition des éléments associatifs; pragmatique, qui vise à influencer et à exciter les sentiments humains.

Il a été constaté que les noms propres, les anglicismes, les chiffres, les onomatopées sonores sont largement utilisés dans les titres des films français. Lors de la traduction, on recourt à des moyens et à des transformations tels que: la traduction littérale, la translittération, la substitution lexico-sémantique, l'addition et l'omission des éléments.

Mots-clés: titre du film, caractéristiques lexicales et sémantiques, culture, moyens de traduction, français, ukrainien.

DOI: https://doi.org/10.23856/4607

\section{Introduction}

La première connaissance du spectateur avec le film commence par son titre. Après l'avoir lu, le spectateur crée son idée et sa première impression de l'œuvre d'art. Ainsi, le succès du film dans le pays de sa distribution, la sympathie du public et le montant dépendent aussi du titre.

Notre étude est consacrée à la problématique de la traduction des titres des longs métrages français en ukrainien. Le cinéma est un loisir très populaire, un phénomène artistique et social qui occupe une place importante dans la vie des gens et fait partie intégrante de la société moderne depuis plus d'un siècle.

Aujourd'hui, les films francophones sont projetés partout dans le monde, notamment en Ukraine. Il existe parfois des problèmes et des difficultés avec la perception et la compréhension de l'information par différentes cultures. C'est pourquoi il y a un besoin de traduction de qualité non seulement du contenu des films eux-mêmes (dialogues, monologues, histoires), mais aussi de leurs titres d'une langue à l'autre. De plus, il est important de transmettre des idées ancrées dans les titres par les cinéastes. 
Plusieurs savants étrangers aussi bien que les linguistes nationaux ont traité le problème de la traduction des titres des films dans leurs recherches. Cependant, un certain nombre de questions a échappé à l'attention des chercheurs, notamment la représentation des valeurs linguistiques et culturelles dans les titres des films français et leur traduction en ukrainien, ce qui détermine l'actualité de notre travail.

Ainsi l'objectif de notre étude est d'établir les moyens de traduction en ukrainien des caractéristiques linguistiques et culturelles des titres de longs métrages français.

Pour atteindre cet objectif, les tâches suivantes sont formulées :

- définir la notion du nom de film et établir ses fonctions principales;

- identifier les caractéristiques lexicales et sémantiques des titres;

- d'établir les stratégies et les moyens utilisés lors de traduction en ukrainien des noms des films français.

Pour une étude approfondie des particularités de traduction en ukrainien des noms de films français ont été utilisées les méthodes suivantes:

- la méthode d'analyse linguistique, à savoir l'analyse de composants pour déterminer la sémantique des noms des films français; l'analyse contextuelle afin d'approfondir l'interprétation significative des modèles identifiés;

- la méthode empirique de généralisation et de classification des résultats de la recherche, leur application dans la pratique;

- la méthode comparative pour identifier les caractéristiques qualitatives et quantitatives des noms de films français et ukrainiens.

\section{Les noms des films et leurs fonctions}

Le titre du film est un type particulier du nom propre, dont l'étude est liée à sa spécificité fonctionnelle, à la définition de son contenu, de son idée, ainsi qu'aux problèmes de sa compréhension et de son interprétation en tant qu'élément principal de la structure informationnelle du film.

Le nom du film est défini comme un énoncé verbalement codée qui représente la situation modélisée par le film (Hromova, 2013: 17). C'est-à-dire qu'il s'agit d'une expression concise de l'idée principale du film.

Afin d'attirer l'attention, les titres des films sont souvent marqués de manière expressive; ils sont brillants, expressifs, ce qui améliore la perception ultérieure du film.

La plupart des linguistes qui avaient étudié les noms des films distinguent leurs cinq fonctions principales: nominative, informative, publicitaire, pronostique et pragmatique (Kazakova, 2002: 49).

Le nom du film remplit une fonction nominative, considérée comme ontologique, puisque le titre est la première étape de la connaissance du spectateur avec le film. La réalisation de cette fonction ne dépend pas des modèles syntaxiques et structurels, ce qui indique l'universalité et l'intégrité de celle-ci (Barhudarov, 1975: 98).

La fonction la plus importante est informative, car le but principal du titre est défini comme la transmission du sens du film afin de préparer le spectateur à ce qu'il verra à l'écran avant de le regarder. En quête d'un film nécessaire ou intéressant pour le spectateur, le dernier fera d'abord attention à son titre (Kazakova, 2002: 53). Cette caractéristique peut être considérée comme dominante, car elle sert de critère principal lors de la création d'un nom du film.

Le rythme de vie accéléré et la sortie d'un grand nombre de films par an, ainsi que le manque constant de temps libre chez l'homme moderne, entraînent une plus grande sélectivité 
et une plus grande attention dans le choix du film. La mise en œuvre de la fonction publicitaire est notée comme la capacité du titre à devenir le plus provocateur et le plus intrigant, grâce à l'utilisation des moyens et des figures stylistiques, des particularités sémantiques et syntaxiques, assurant ainsi le succès du film et en attirant plus de spectateurs. La fonction publicitaire est responsable de la formation d'une certaine perception chez le public, retenant l'attention et renforçant le sentiment d'attente (Hromova, 2013: 97).

La fonction pronostique est considérée comme un processus d'anticipation, c'est-à-dire un mécanisme mental complexe de prédiction par le spectateur du contenu du film d'après son titre. Le spectateur prédit, formule et construit consciemment ou inconsciemment dans son imagination le contenu du film caché dans le titre. Au cours de l'anticipation, le spectateur s'appuie sur l'expérience individuelle, le lexique, ainsi que sur les connaissances générales de base.

La fonction pronostique est réalisée par l'adaptation sémantique c'est-à-dire en ajoutant des mots associatifs qui relient le titre au genre et au sujet du film. Les noms des films doivent être relativement simples et faciles à retenir.

La fonction pragmatique est comprise comme l'utilisation du langage pour une influence intellectuelle, émotionnelle ou volontaire sur le spectateur potentiel (Arkhypova, 2002: 89). Le nom du film forme la perception du spectateur et provoque généralement chez lui curiosité, surprise, indignation, confusion, déception etc.

Ainsi l'équivalence, l'adéquation, l'adaptation, la concision et bien d'autres aspects déterminent certaines caractéristiques et fonctions des noms de films et nécessitent une approche particulière de leur traduction.

\section{Caractéristiques lexico-sémantiques des noms des films sous l'aspect de traduction}

L'approche linguo-culturelle joue un rôle très important dans le processus de traduction des titres des films, car l'accent mis sur la culture détermine le choix de certains moyens de traduction, tels que l'omission ou le remplacement des éléments du titre, s'ils sont inacceptables dans cette culture pour des raisons idéologiques, esthétiques ou morales ou ne correspondent pas correctement aux associations par rapport à la culture de la source.

Le choix des moyens de traduction des noms des films français en ukrainien est déterminé par les caractéristiques lexicales et sémantiques que nous avons identifiées, telles que :

1) la présence des noms propores;

2) l'emploi des anglicismes;

3) l'utilisation des chiffres quantitatifs;

4) l'emploi des onomatopées sonores.

Les recherches montrent que lors de la traduction, les spécialistes ont souvent recours à des moyens et à des transformations pour une traduction adéquate, telles que la traduction littérale, la translittération, la substitution lexico-sémantique, l'addition ou l'omission des éléments, l'adaptation, etc.

Un exemple d'utilisation de la traduction littérale peut être les noms de films suivants, dans la traduction desquels la correspondance lexicale et sémantique de l'original a été conservée: «Les vacances du petit Nicolas» - «Канікули маленького Ніколя»;

«Mon meilleur ami» - «Mій найкраший друг»;

«La famille Bélier» - «Ciм'я Бельє»;

«Mon rоi»- «Мій король».

A.B. Kamenets exprime l'opinion que la traduction littérale des titres est utilisée en l'absence de réalités socioculturelles intraduisibles et du conflit entre la forme et le contenu 
(Kamianets, 2007: 164). Nous devrions réfléchir à cet axiome. L'analyse des titres originaux français et de leurs versions ukrainiennes traduites montre que dans certains cas les titres sont intégralement conservés, par exemple:

«Olé!»-«Оле!»;

«Qui?»- «Xmo?»;

«La bouche de Jean-Pierre» - «Губи Жан-П’єра».

Dans d'autres cas les titres subissent des transformations partielles ou radicales lors de la traduction. Par exemple:

«Quand l'amour s'emmêle» - «Коли втручається любов»;

«Les garçons et Guillaume, à table!» - «Я, знову я і мама»;

«Amour \& turbulences»- «Кохання без пересадок».

Le deuxième moyen de traduction des noms est défini comme la translittération, lorsqu'il y a un transfert précis des signes d'une écriture par les signes d'une autre, dans laquelle chaque symbole d'un système d'écriture est transmis par le signe correspondant d'un autre système d'écriture (Batiushchenko, 2015: 94). Ce moyen de traduction est également utilisé pour les noms de films avec le nom propre du héros. Habituellement les noms propres peuvent être transmis par translittération (au niveau de la graphie) ou transcription (au niveau des phonèmes). Des exemples des noms des films sont les suivants :

«Paris-Manhattan» - «Париж-Манхеттен»;

«Les nouvelles aventures d'Aladin» - «Нові пригоди Ала,ддіна»;

«Marguerite»- «Mapzapuma»;

«Coco avant Chanel» - «Коко до Шанель»;

«Les frères Sisters» - «Брати Cicmepc».

Lors de la traduction des titres, les noms uniques sont entièrement conservés par translittération. Les noms mélodiques et beaux sont dotés de magnétisme, attirent l'attention. Les titres de films sont intéressants et faciles à retenir. Les films avec des noms propres dans les titres sont attrayants car un personnage brillant, original et remarquable apparaît dans l'imagination du spectateur.

Un autre moyen de traduction des noms de films est la substitution, qui peut être due à des raisons à la fois linguistiques et extralinguistiques. Les parties du discours, les formes des mots, les structures syntaxiques d'une phrase, le sens des mots peuvent être remplacées. Cependant, quels que soient les remplacements effectués par le traducteur, ils doivent toujours être motivés et ne doivent pas conduire à un changement dans la structure pragmatique de l'énoncé et du texte dans son ensemble, violation des normes de communication linguistique dans la langue de traduction (Komissarov, 2001: 153). Ce moyen de traduction est assez courant, il inclut des substitutions de type général, c'est-à-dire lexical, ainsi que la concretisation, la généralisation et d'autres.

Par exemple, dans le titre suivant «Les petits тоисhoirs»- «Маленькі секрети» en cas de la traduction littérale du mot mоисhoir comme хустинка le sens principal du film se perd, car il s'agit des secrets qui ont été racontés en compagnie d'amis.

Le film «Les émotifs anonymes» raconte une histoire de deux jeunes amoureux timides qui tentent de surmonter leur peur et d'arranger la vie personnelle. La traduction littérale du mot émotif ne représente pas suffisamment le message principal du film, c'est pourquoi les traducteurs ont utilisé la concrétisation en le traduisant comme "Анонімні романтики».

Le moyen suivant utilisé dans la traduction des noms de films consiste en divers additions, c'est-à-dire l'élargissement du texte de la langue cible par rapport au texte original, motivé par des raisons linguistiques et extralinguistiques (Komissarov, 2001: 158). Ces éléments de 
traduction servent souvent à révéler mieux l'intrigue, indiquant le genre du film. De brefs commentaires supplémentaires sur le titre original français donnent au public un certain ton émotionnel. On estime que la traduction qui élargit le concept est causée par le désir du traducteur de fournir plus d'informations ou de luminosité. Il est possible que les traducteurs considèrent qu'il est important d'introduire des mots-clés dans le titre ukrainien afin de combler l'insuffisance sémantique et de genre du titre original.

Ainsi, lors de la traduction du titre français «Knоck» comme «Афера доктора Нока» on a utilisé la transformation consistant à ajouter des éléments lexicaux afin d'intéresser les spectateurs potentiels. Etant donné que le nom Knock lui-même n'est pas suffisamment informatif pour attirer l'attention des gens, l'addition d'une profession (médecin) et le mot «aфepa» a un effet pragmatique important sur le public et crée une intrigue.

Citons encore quelques exemples plus frappants de l'application de cette transformation en traduction :

«Saint Laurent»- «Сен-Лоран. Стиль - ие я»;

«Dalida» - «Любов і пристрасть. Даліда»;

«Victoria» - «У ліжку з Вікторією» ;

«Fanfan»- «Аромат кохання. Фанфан».

Un autre moyen courant de traduction des noms des films français est l'omission. Les raisons d'utiliser cette transformation, opposée à l'addition et aussi appelée comme «compression», peuvent être linguistiques et extralinguistiques (Komissarov, 2001: 159).

Le titre français «Le Fabuleux Destin d'Amélie Poulain» signifie littéralement «Казкова доля Амелі Пулен». Dans la distribution ukrainienne, les spectateurs ont regardé ce film intitulé "Амелі». Le traducteur a prédit qu'un nom attrayant français dans le titre suffirait à distinguer le film des autres, et qu'il serait facile à retenir.

Un autre exemple illustre est le film «Les deux amis» - «Друзі», où le traducteur a omis le chiffre deux, estimant que le film peut recevoir un nom plus général. C'était peut-être une allusion à la célèbre série populaire «Друзі», dont la mention devrait faire des certaines associations et intéresser les spectateurs.

Il est à noter qu'il y a des titres qui ne correspondent pas dans la langue cible à l'original. Il s'agit de la traduction libre sans tenir compte des composantes formelles et sémantiques du texte source (Korobova, 1994: 39). Cette technique s'explique par le fait que le titre remplit en traduction certaines fonctions pragmatiques, dont la réalisation exige l'emploi des moyens linguistiques appropriés. Les noms de films suivants montrent cette approche de traduction. Par exemple, «Lolo»- «Матусин синок».

Dans ce film, Lolo est le personnage principal, qui est très attaché à sa mère et contrôle sa vie personnelle. L'expression «матусин синок» dans la culture ukrainienne est péjorative, et ce cas ne fait pas exception. Ainsi, c'est pour la réalisation de l'effet pragmatique que la transformation de l'adaptation a été utilisée.

Dans certains cas, l'adaptation provoque une transformation complète des noms propres des héros dans les titres français, par exemple :

«Ombline» - «Я стану краще».

Dans le drame psychologique «Ombline» de S. Kaza (littéralement «Омблін»), on raconte au spectateur les situations de vie difficiles d'Ombline, qui a donné naissance à un fils en prison. Pour être avec un enfant, une fille veut sortir de prison. Le titre ukrainien « кращ̧е» non seulement ne déforme pas l'essence du film, mais, au contraire, est une sorte de clé pour le comprendre. 
L'exemple suivant est le titre de la comédie de Jean Junio, qui raconte les aventures du drôle de personnage Budu: «Воиdи» - «Щастить, як потопельникові». Une telle traduction ironique du titre dans la distibution ukrainienne forme une certaine image du film, promet au spectateur un passe-temps facile et amusant.

L'histoire biographique intitulée «Victor Young Perez» raconte le destin tragique du célèbre Français Victor Perez - un boxeur, champion du monde, qui a vécu à Paris avant la Seconde Guerre mondiale et a été tué dans un camp de concentration. Le nom de Victor Perez est inconnu pour la majorité de la population ukrainienne. Cette histoire avec une traduction littérale de «Віктор Янг Перез» ne serait plus attrayante que pour le public français. Par conséquent, le titre de ce film dans la traduction ukrainienne est «Жорстокий ринг», се qui attire l'attention des cinéphiles - les fans de combats, d'arts martiaux et d'effets spéciaux.

Les traducteurs connaissaient bien les intrigues de ces peintures, changeant complètement les noms français et considérant leurs propres versions plus avantageuses. Il est évident que le succès du film auprès du public dépend du titre bien choisi. On pense parfois qu'un changement radical du titre, allant jusqu'à la création d'un nouveau, est nécessaire pour attirer l'attention du spectateur sur le visionnage du film, pour l'intriguer. Les professionnels hautement qualifiés n'ont pas peur d'expérimenter avec le texte et sont constamment à la recherche de nouvelles façons d'attirer l'attention du public. Ainsi, la comédie française «Rien à déclarеr» dans la distribution nationale s'appelait «Митниия дає добро», alors que le titre se traduit littéralement comme «Нічого декларувати».

La comédie se moque de l'inimitié locale entre les douaniers français et belges. Le nom français «Rien à déclarer» est utilisé de manière symbolique et ironique, prédisant le genre et l'intrigue du film. Pour adapter le titre, une citation préférée de tous, légère et bonne a été tirée de la comédie soviétique «Біле сонце пустелі». Une indication directe de celle-ci promet bonne humeur et plaisir lors du visionnage d'un film français. Ce qui était important pour le traducteur c'était une phrase symbolique dans l'espace culturel ukrainien. Pour inspirer confiance et séduire le public, les traducteurs ont pris en compte les passions et la mentalité de l'auditoire national.

Une autre comédie «Un heureux événement» lors de l'adaptation a reçu le nom badin dans la distribution ukrainienne «Сексу багато не буває». Le titre français attire l'attention du spectateur sur les problèmes survenus dans une jeune famille après un événement heureux - la naissance d'un enfant. Le nom français est ironique, puisque d'après le sujet la naissance d'un enfant a causé des problèmes et des conflits dans le couple. Les distributeurs de films ukrainiens ont décidé de remplacer complètement le nom français en choisissant le titre ukrainien plein d'esprit qui évoque involontairement des expressions telles que «грошей багато не буває», «горілки багато не буває» еtс.

Le mélodrame intitulé «Mes chères études» a été présenté au public ukrainien sous le titre «Студентка за викликом». Un titre audacieux destiné à un jeune public dicte au spectateur l'intrigue du film.

Les versions de la traduction des noms des films présentées ci-dessus sont attrayantes. Ils affectent les sentiments et les émotions du public, encouragent le visionnage. Cependant, il $\mathrm{y}$ a de fortes critiques des cas où les promoteurs ukrainiens déforment délibérément et déraisonnablement le titre pour rendre l'image plus réussie dans la distribution. Ainsi la comédie dramatique française «Intouchables» est sortie en Ukraine sous le titre de « $1+1 »$. Une histoire intéressante raconte une amitié entre un aristocrate handicapé et son assistant noir, joué par l'acteur Omar Si. En Ukraine ce film a eu un énorme succès. 
L'une des interprétations de «l'inviolabilité» des personnages principaux du film est que les personnes handicapées et à la peau noire semblent être «inviolables», car dans une société tolérante on communique avec elles avec beaucoup de prudence, de peur les offenser. Une traduction littérale de ce titre, à notre avis, conserverait son originalité et présentererait l'idée originale des réalisateurs, étant plus pertinente que la traduction ukrainienne.

Donc, on peut affirmer qu'il n'y a pas de titre parfait, qu'il n'y a pas non plus d'une seule adaptation correcte en langue étrangère. Le titre du film et sa traduction sont déterminés par des facteurs culturels et linguistiques, et leur perception est purement individuelle. La reconnaissance ou la désapprobation des titres sont généralement causées par les préférences personnelles des spectateurs et des critiques de cinéma.

Comme exemple, prenons le titre d'une comédie «Sa majesté Minor» qui porte le nom dans la distribution ukrainienne «Мільйон років до намої ери 2». Malgré le titre ce film n'est pas une suite du film «Мільйон років до намої ери». Il s'agit d'une autre comédie satirique tournée par un autre réalisateur. Les spectateurs attirés par l'analogie fictive du titre, ont été indignés et bouleversés par le «faux» nom. Cependant l'intrigue du film lui-même a provoqué une discussion animée parmi les critiques de cinéma et les cinéphiles. Des avis contradictoires ont affecté un grand nombre de scènes de sexe, de la bonne musique, un jeu d'acteur, des références à des mythes anciens.

Lors de visionnage du film le spectateur percevra ce qui se passe à l'écran à travers le prisme d'un titre intéressant. Un nom inhabituel restera dans la mémoire du spectateur, puis sera facilement restauré et reproduit. L'un des titres qu'on peut considérer comme réussi pour attirer un large public est le titre comprenant des chiffres. Par exemple:

«3 сеиин»- - 3 серия»;

«99 francs» - «99 франків»;

«5x2»- «5x2»;

«8 fеттеs»- «8 жінок».

Les titres pareils ne sont pas typiques pour la distribution de films ukrainiens et suscitent un intérêt supplémentaire chez les spectateurs potentiels.

\section{Conclusion}

Ainsi la tâche des titres français est de maximiser la transmission du sens et de l'idée générale du film et d'attirer l'attention du public cible. Les noms des films français peuvent être courts et longs, mais ils sont toujours spectaculaires et intrigants. Des noms propores, des anglicismes, des chiffres, des onomatopées sonores et de courtes phrases mystérieuses sont souvent utilisés. Quant à leur traduction, c'est souvent le recours à l'adaptation pour refléter les réalités de culture.

De plus, à une époque de forte concurrence, il est important non seulement de créer une traduction équivalente au titre original, mais aussi de le rendre attractive pour le public cible. Les noms de films français traduits en ukrainien conservent leur qualité. Les titres transformés lors de traduction affectent l'esprit de l'auditoire potentiel, évoquant divers types d'associations.

Lors de la traduction, l'un des grands principes est le niveau d'équivalence de l'original. Dans la plupart des cas, le titre dans la traduction et l'original se correspondent, mais il existe des situations où il est nécessaire de s'écarter de ce principe compte tenu des spécificités nationales et culturelles de la langue et des connaissances de base de ses représentant. Afin de traduire adéquatement les noms de films français en ukrainien, des moyens et des transformations 
tels que la traduction littérale, la translittération, la substitution lexico-sémantique, l'addition ou l'omission des éléments, l'adaptation ont été utilisés. Ainsi, le problème de la traduction des noms de films n'est pas seulement linguistique mais aussi culturel et moral.

Une piste de recherche ultérieure consistera à déterminer plus précisément les aspects concernant les particularités grammaticales et stylistiques de traduction en ukrainien des noms des films français.

\section{Littérature}

Arkhypova, L.V. (2002). Pereklad yak interpretatsiia. Zapysky Perekladatskoi Maisterni [Translation as interpretation. Notes of the Translation Workshop]. Lviv: Yaremcha. [in Ukrainian].

Barhudarov L.S. (1975). Yazyk i perevod (Voprosy obshej i chastnoj teorii perevoda) [Language and translation (Questions of general and particular theory of translation)]. Moscow: Mezhdunarodnye otnosheniya. [in Russian].

Batiushchenko Ye.I. (2015). Poniattia filmonimu v konteksti teorii onomastyky [The concept of film name in the context of the theory of onomastics]. Lviv: Ukrainska akademiia drukarstva. [in Ukrainian].

Hromova Z.V. (2013). Osnovni pomylky pry perekladi nazv kinofilmiv [Main mistakes when translating movie titles]. Bulletin of Luhansk Taras Shevchenko National University. Philological sciences, vol. 9(1). [in Ukrainian].

Kamianets A.B. (2007). Pro kryterii adekvatnosti khudozhnoho perekladu. Mova i kultura [On the criteria of adequacy of literary translation. Language and culture]. Bulletin of Taras Shevchenko National University of Kyiv, vol.41. [in Ukrainian].

Kazakova T.A. (2002). Prakticheskie osnovy perevoda [Practical translation basics]. Moscow: Soyuz. [in Russian].

Komissarov V.N. (2001). Sovremennoe perevodovedenie [Modern translation studies]. Moscow: ETS. [in Russian].

Korobova L.A. (1994). Zaglavie kak komponent teksta [Title as a text component]. Moscow: ETS. [in Russian]. 